study, adolescents were asked to complete the A-CHAQ and $\mathrm{HAQ}$ and their guardians the P-CHAQ. Adolescents were selected if at least two of the questionnaires had been completed simultaneously within the first year following diagnosis.

Fit of the item response models was assessed by comparing model expected item scores with those observed in CAPS for each item (i.e. residuals). An item response model was considered to adequately describe item response behaviour of CAPS patients when the mean of the residuals was $< \pm 5 \%$. Agreement of overall questionnaire IRT scores were then compared using limits of agreement (2SD) and intra-class correlations.

Results: Of 303 adolescents, $61 \%$ were female and median age at JIA diagnosis was 13 years (range 11 to 17). Raw HAQ scores consistently fell below both $\mathrm{CHAQ}$ scores.

IRT model fit in the CAPS population was good, with $1 \%$ of item residuals $> \pm 5 \%$. When modelled using IRT, the mean differences in overall scores approximated zero, with narrow limits of agreement, at 15 (PCHAQ vs $A C H A Q), 12$ (PCHAQ vs $\mathrm{HAQ}$ ) and 10 (ACHAQ vs HAQ), on a 0-100 scale. High intra-class correlations between overall scores were evident (range 0.83 to 0.90 ). There was therefore high agreement between IRT-modelled scores obtained for different questionnaires. A scale characteristic curve (figure 1) illustrates the relationship between the expected scores for $\mathrm{CHAQ}$ and $\mathrm{HAQ}$ questionnaires with the latent functional ability variable.

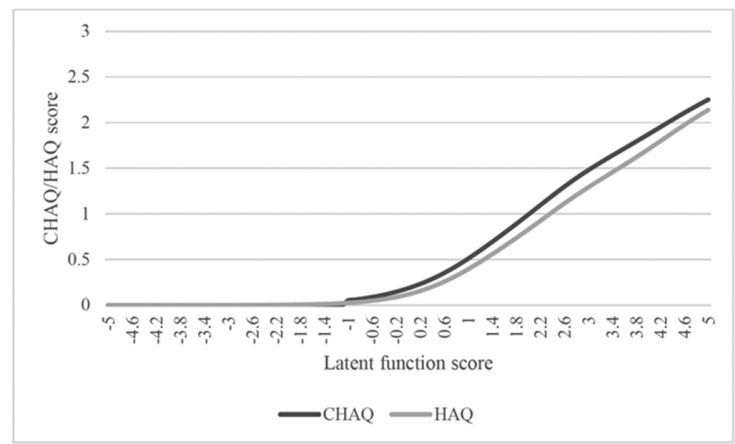

Figure 1 Camparing $\mathrm{CHAQ}$ and $\mathrm{HAQ}$ scores with latent function.

Conclusions: IRT models for functional ability previously developed in a mixed population of adult and paediatric patients with inflammatory arthritides are applicable to adolescents with JIA in CAPS. IRT scores across CHAQ and HAQ measures had high agreement. IRT scores for functional ability can therefore be used in clinical practice and research to directly compare scores on the CHAQ and HAQ. This will be important as adolescents transfer from paediatric to adult rheumatology.

Disclosure of Interest: None declared

DOI: 10.1136/annrheumdis-2018-eular.3670

\section{FRI0649 SYSTEMIC LEVELS OF TYPE VI COLLAGEN METABOLITES ARE ELEVATED IN RA PATIENTS AND MODULATED BY TREATMENT WITH ANTI-IL6R THERAPY}

C. S. Thudium ${ }^{1}$, N. S. Gudmann ${ }^{1}$, M. Karsdal ${ }^{1}$, A.-C. Bay-Jensen ${ }^{1} .{ }^{1}$ Biomarkers \& Research, Nordic Bioscience, Herlev, Denmark

Background: Rheumatoid arthritis (RA) is a chronic autoimmune disease characterized by progressive inflammation systemically and local joint deterioration. Chronic inflammation leads to elevated levels of tissue remodeling and release of extracellular matrix (ECM) metabolites into circulation. Type $\mathrm{VI}$ collagen (Col-VI) is found at the interface between the interstitial- and the basement membrane where it binds other matrix proteins and support cell-cell interactions. Blood levels of Col-VI metabolites (C6M) have been associated with increased tissue turnover and disease activity in animal models and clinical studies of lung ${ }^{1}$ and liver ${ }^{2}$ fibrosis and ankylosing spondylitis ${ }^{3}$. These studies indicate that systemic levels of $\mathrm{C} 6 \mathrm{M}$ are released ubiquitously from many organs and may be modulated by antiinflammatory treatments. Treatment with the $\alpha \mathrm{IL}-6$ receptor agent Tocilizumab (TCZ) results in decreased ECM remodeling

Objectives: Investigate the modulation of Col-VI metabolites in circulation by TCZ treatment and its association with treatment response.

Methods: Systemic Col-VI metabolism was measured in the LITHE study $(n=740)$, a one year double blind, placebo controlled phase III parallel group study in patients receiving placebo, $4 \mathrm{mg} / \mathrm{kg}$ or $8 \mathrm{mg} / \mathrm{kg}$ TCZ in combination with MTX. Col-VI degradation was measured using the $\mathrm{C} 6 \mathrm{M}$ assay, measuring a specific MMP generated metabolite at bl, w4 and w16. The odds ratio (OR) of treatment response (ACR20, 50 or 70) at >median decrease was calculated using logistic regression, adjusting for age, sex, BMI, dis. duration (model 1) and treatment (model 2).

Results: TCZ treatment dose dependently reduced of Col-VI metabolites in serum at w4 in both 4 and $8 \mathrm{mg} / \mathrm{kg}(9 \% \mathrm{~ns}$ and $46 \% \mathrm{p}<0.0001)$ doses compared to placebo and the reduction persisted throughout the study. Patients with a high suppression of Col-VI turnover were more likely to respond to treatment compared to patients with low suppression. In patients with high suppression after 4 weeks the OR and ACR20 response at w16 was $1.6(95 \% \mathrm{Cl}, 1.16-2.20)$, although this was not significant when adjusting for treatment. The OR of ACR50 was $2.2(95 \%$ $\mathrm{Cl}, 1.507-3.296)$ and for ACR70 2.6 (95\% Cl, 1.407-4.631).

Table 1 Association of high/low C6M median \% change with ACR response after 16 weeks using logistic regression

\begin{tabular}{lllllllll}
\hline & & Outcome & ACR20 & & ACR50 & \multicolumn{2}{l}{ ACR70 } & \\
\hline Determinant & Model & & OR & p- & OR & p-value & OR & p- \\
& & & $(95 \%$ & value & $(95 \%$ & & $(95 \%$ & value \\
& & & $\mathrm{Cl})$ & & $\mathrm{Cl})$ & $\mathrm{Cl})$ & \\
\hline $\mathbf{\Delta \text { C6M \% }}$ & Model & High & $\mathbf{1 . 5 9 6}$ & 0.004 & $\mathbf{2 . 2 2 8}$ & $<0.001$ & $\mathbf{2 . 5 5 3}$ & 0.002 \\
week 4 & $\mathbf{1}$ & & $(1.160-$ & & $(1.507-$ & & $(1.407-$ & \\
& & & $2.198)$ & & $3.296)$ & & $4.631)$ & \\
& Model & High & 1.319 & 0.122 & $\mathbf{1 . 8 2 0}$ & 0.005 & $\mathbf{2 . 3 9 0}$ & 0.007 \\
& 2 & & $(0.929-$ & & $(1.189-$ & & $(1.265-$ & \\
& & & $1.873)$ & & $2.785)$ & & $4.517)$ & \\
\hline
\end{tabular}

Conclusions: Suppression of Col-VI turnover was significantly associated with a higher level of response to TCZ treatment in RA patients after already 4 weeks We hypothesize that there is an increased systemic connective tissue turnover in RA and connective tissue metabolites may be used as indicators of treatment response.

\section{REFERENCES}

1. Jenkins RG, Simpson JK, Saini G, et al. Lancet Respir Med. 2015;2600 (15):1-11.

2. Leeming DJ, Byrjalsen I, Jiménez W, Christiansen C, Karsdal MA, Liver Int. 2013;33(3):439-447.

3. Bay-Jensen AC, Leeming DJ, Kleyer A, Veidal SS, Schett G, Karsdal MA. Rheumatol Int. 2012;32(11):3565-3572.

Disclosure of Interest: C. Thudium Employee of: Nordic Bioscience, N. Gudmann Employee of: Nordic Bioscience, M. Karsdal Shareholder of: Nordic Bio science, Employee of: Nordic Bioscience, A.-C. Bay-Jensen: None declared DOI: 10.1136/annrheumdis-2018-eular.6823

\section{FRI0650 THE DIAGNOSTIC VALUE OF THE AESKULISA PR3 SENSITIVE \& AESKULISA MPO IN THE EUVAS-COHORT}

K. Prager ${ }^{1}$, N. Marte ${ }^{1}$, E. Csernok ${ }^{2}$, A. Lerner ${ }^{3}$, T. Matthias ${ }^{3} .{ }^{1} R \& D$, Aesku. Diagnostics GmbH \& Co. KG, Wendelsheim, ${ }^{2}$ Department of Internal Medicine, Vasculitis-Center Tübingen-Kirchheim, Medius Klinik Kirchheim, Kirchheim-Teck, ${ }^{3} R \& D$, Aesku.kipp Institute, Wendelsheim, Germany

Background: Anti-neutrophil-cytoplasmic-antibodies directed against proteinase-3 (PR3-ANCA) and myeloperoxidase (MPO-ANCA) are serological hallmarks of small vessel vasculitis, particularly granulomatosis with polyangiitis (GPA) and microscopic polyangiitis (MPA). In a recent multicentre European-Vasculitis-Study-Group (EUVAS) evaluation, the performance of IIF was compared to that of various antigen-specific immunoassays.

Objectives: The aim was to evaluate the diagnostic accuracy of the third-generation antigen-specific immunoassays PR3-ANCA (AESKULISA-PR3-sensitive) and MPO-ANCA (AESKULISA-MPO) and to compare these data with the results from the other assay (Orgentec).

Methods: 257 samples from the EUVAS cohort were tested for the presence of ANCA by PR3-ANCA ELISA (AESKULISA-PR3-sensitive) and MPO-ANCA ELISA (AESKULISA-MPO). Newly diagnosed GPA/MPA $(n=66)$ patients and diseased controls $(n=191)$ : systemic lupus erythematosus $(n=60)$, systemic sclerosis $(n=10)$, rheumatoid arthritis $(n=90)$, Scleroderma $(n=11)$ and Sjögren's syndrome $(n=30)$ were analyzed.

Results: In AAV patients, ANCAs were detected with both methods in 56 cases; divergent results were obtained in only 1 patient sample. 191 patients with othe rheumatic diseases were analyzed and only 13 vs 11 (AESKU/Orgentec) were 